(5) photographic observations of a spot in the ranges $\lambda \lambda 5725-5840$ and $\lambda \lambda 6610-6770$ obtained by $O$. Engvold at Oslo.

Item (2) is available on magnetic tape; (3) is soon to be published on a roll of print paper $100 \mathrm{~m}$ long; the first part of item (4), 1 to $2.5 \mu \mathrm{m}$, has been prepared as an atlas and is in press - future work by Hall will make available the atlas' extension to $12 \mu \mathrm{m}$.

The large amount of work on the identification of molecular lines and species will only be quoted by two references from which many other references can be found:

'On Molecules in Sunspots', H. Wöhl, Solar Phys. 16, 362, 1971.

'Table of Solar Diatomic Molecular Lines Spectral Range 4900-6441 A', P. Sotirovski, Astron. and Astrophys. Suppl. Ser. 6, 85, 1972.

A. KEITH PIER CE

Chairman of the Working Group

\title{
WORKING GROUP ON SOLAR ECLIPSES
}

The total solar eclipse of July 10,1972, was observed by several parties and it is expected that reports on the observations will be given during the 1973 IAU General Assembly. All information required was provided by $\mathrm{Dr}$ V. Gaizauskas and other Canadian colleagues and distributed to interested persons.

Much work has been done in view of the difficulties the observing groups in Africa will have to overcome before, during, and after the eclipse of June 30, 1973. The National Science Foundation has published a Solar Eclipse 1973 Bulletin, as it did for the eclipse of March 7, 1970. The N.S.F. co-ordinator, Dr Ronald R. La Count, has distributed the bulletin widely, reporting on site inspections, meteorological forecasts, general information, relevant data and details on the circumstances of the eclipse.

The joint efforts of Dr E. R. Dyer, Jr., secretary of the IUCSTP, Dr Ronald R. LaCount, Prof. C. de Jager, and those of the Working Group have led, among other things, to the organization and scientific programs connected with the 1973 eclipse. Two meetings were held during the 15th and 16th Annual Meetings of COSPAR in Seattle and Madrid, and a third during the first IAU Regional meeting in Athens. African authorities invited by the General Secretary of the IAU attended the Madrid meeting and promised assistance to observing parties and help for the general organization.

According to a resolution of the Working Group on Solar Eclipses, the Chairman asked the General Secretary to propose a member of the group as a representative to COSPAR. Dr J. Houtgast, former chairman of the Group, has been appointed.

M. RIGUTTI

Chairman of the Working Group

\section{REFERENCES}

\section{Legend}

AA Astronomy \& Astrophys.

AiA

AJ

AL

AMZ

AO

ARAA

ASHSN

AT

ATAO

AZ
Astrometriya i Astrophysica

Astrophys. Journal

Astrophysics Letters

Astr. mitt Zürich

Applied Optics

Ann. Rev. Astron. Astrophys.

Actes Soc. Helv. Sci. Nat.

Astron. Tsirk

Ann. Tokyo Astron. Obs.

Astron. Zhurn. Akad. Nauk S.S.S.R.
BAC

BAAS

BAOM

GGV

IKAO

IAU

JASWA

JGR

JQSRT

MAG

MN

MSRSL
Bull. Astron. Inst. Czechoslovakia Bull. American Astron. Soc. Bol. Astron. Obs. Madrid Geod. Geophys. Viroff Izv. Krimskoj Astrofiz Obs. IAU Symposium

J. Astron. Soc. Western Australia J. Geophys. Research

J. Quant. Spectrosc. Radiat. Transfer Mitt. Astron. Ges. Mon. Not. Roy. Astr. Soc. Mem. Soc. Roy. Sciences de Liége 


$\begin{array}{ll}\text { N } & \text { Nature } \\ \text { NPS } & \text { Nature Phys. Sci. } \\ \text { P } & \text { In press } \\ \text { PASA } & \text { Proc. Astr. Soc. Australia } \\ \text { PASJ } & \text { Publ. Astr. Soc. Japan } \\ \text { PNAS } & \text { Proc. Nat. Academy of Science USA } \\ \text { PRL } & \text { Phys. Rev. Letters } \\ \text { PTRSL } & \text { Phil. Trans. Roy. Soc. London } \\ \text { SAAJ } & \text { Soviet Astron. AJ } \\ \text { SDB } & \text { Solnechnye Dannye Byull. } \\ \text { SP } & \text { Solar Physics } \\ \text { SSR } & \text { Space Science Reviews } \\ \text { U } & \text { Unpublished } \\ \text { VANS } & \text { Vest. Akad. Nauk S.S.S.R. } \\ \text { VSNG } & \text { Verh. Schweiz. Naturf. Ges. } \\ \text { ZA } & \text { Zeits. Astrophys. }\end{array}$

Alikayeva, Bekchantayeva (1972) AiA 17

Alissandrakis, Macris (1971) SP 20, 47

Altrock, Canfield (1972a) SP 23, 257

Altrock, Canfield (1972b) AJ 171, L71

Altschuler, Newkirk (1969) SP 9, 131

Altschuler, Perry (1972) SP 23, 410

Altschuler et al. (1972) SP (P)

Applied Optics (1970) 9 (No. 12)

Arnquist, Menzel (1970) SP 11, 82

Arroyo, Torrecilla (1971) BAOM 7, 5

Athay (1970) SP 11, 237

Athay (1971) Physics of the Solar Corona, Reidel, Dordrecht, p. 35

Athay, Canfield (1970) NBS Spec. Publ. 332, 65

Athay et al. (1972) SP 24, 18

Axisa et al. (1971) SP 19, 110

Badalyan (1971) AT 622, 3

Badalyan, Livshitz (1972) SP 22, 297

Baluteau (1971) AA 14, 428

Banos, Macris (1970) SP 12, 106

Bappu, Sivaraman (1971) SP 17, 316

Bappu et al. (1972) SP (P)

Baschek et al. (1970) AA 4, 229

Batstone et al. (1970) SP 13, 289

Beckers (1968) SP 3, 367

Beckers (1971) IAU 43, 3

Beckers (1972) ARAA 10, 73

Beckers, Morrison (1970) SP 14, 280

Beckers, Schroter (1968) SP 4, 142

Beckers, Wagner (1971) SP 21, 439

Beckers et al. (P)

Bell (1972) BAAS 4, 378

Bhatnagar, Tanaka (1972) SP 24, 87

Bhattacharyya (1972) SP 24, 274

Billings (1970) SP 14, 168

Billings, Oh (1971) SP 21, 418

Blackwell et al. (1972) SP 23, 292

Blaha (1971) SP 17, 99

Blumenthal et al. (1972) AJ 172, 205
Bohlin (1970a) SP 12, 240

Bohlin (1970b) SP 13, 153

Bohlin (1971) SP 18, 450

Bohlin, Garrison (1972) BAAS 4, 378

Bohlin et al. (1971) SP 21, 408

Boland et al. (1971 a) 17, 333

Boland et al. (1971b) PTRSL A270, 29

Boland et al. (1971c) PTRSL A270, 47

Boland et al. AA (P)

Bonnet (1968) AA 31, 597

Bonnet, Blamont (1968) SP 3, 64

Boyer et al. (1971) SP 19, 330

Boyle (1972) BAAS 4, 268

Brault (1972) Auxiliary Instrumentation for Large Telescopes, CERN Conf. Proc., 367

Brauninger et al. (1971) SP 20, 81

Bray (1969) SP 10,63

Bray, Loughhead, The Solar Chapman \& Hall, London (P)

Bray, Winter (1970) SP 15, 309

Bridges, Wiese (1970) AJ 161, L71

Brueckner (1971) AJ 169, 621

Bruzek, De Mastus (1970) SP 12, 447

Burger, Dijkstra (1972) SP 24, 395

Burton, W. Ridgely (1970) SP 14, 3

Burton C. et al. (1971) PTRSL A270, 81

Byard, Kissels (1971) SP 21, 351

Caccin et al. (1970) SP 13, 33

Caccin et al. (1971) SP 17, 89

Canfield (1971) AA 10, 64

Catura et al. (1972) BAAS 4, 379

Chambe (1971) AA 12, 210

Chapman (1970) SP 13, 78

Chevalier, Lambert (1970) SP 11, 243

Clark et al. (1971) PTRSL A270, 55

Cooper et al. (1971) JQSRT 11, 263

Cowan, Widing (1972) BAAS 4, 380

Cox, Tucker (1969) AJ 157, 1157

Cram (1972) SP 22, 375.

Culhane et al. (1970) SP 15, 394

Cuny (1971) SP 16, 293

Curtis et al. (1971) NCAR Ann. Rept. 127

David (1961) ZA 53, 37

Debarbat et al. (1970a) AA 8, 231

Debarbat et al. (1970b) AL 6, 251

De Boer, Pottasch (1972) SP 23, 406

De Boer et al. (1972) AA 16, 417

Deinzer (1971) MAG 30, 67

de Jager, Neven (1972) SP 22, 49

Deubner (1971) SP 17, 6

Dollfus (1971) Physics of the Solar Corona, Reidel, Dordrecht, 97

Drago (1970) SP 13, 357

Dubov (1917) SP 18, 43

Dulk, Altschuler (1971) SP 20, 438

Dulk et al. (1971) AL 8, 235 
Dumont, Pecker (1971) AA 10, 118

Dunn, Zirker (1972) BAAS 4, 381

Dupree (1972) BAAS 4, 381

Dupree, Reeves (1971) AJ 165, 599

Durasova et al. (1971) NPS 229, 82

Durrant, Michalitsanos (1971) SP 18, 60

Dyer (1972) Solar-Terrestrial Physics 1970, Reidel, Dordrecht

Eddy (1972) BAAS 4, 382

Eddy, Goff (1971) BAAS 3, 261

Edlén (1972) SP 24, 356

Edmonds (1962) AJ Suppl. 6, 357

Edmonds et al. (1971) BAAS 3, 6

Edmonds et al. (1972) SP 23, 47

Elste (1967) AJ 148, 857

Elste, Hartoog (1972) in prep.

Evans (1970) SP 14, 157

Fainberg, Stone (1971) SP 17, 392

Fay, Wyller (1970) SP 11, 384

Finn (1972) AA (P)

Fisher (1971a) SP 16, 111

Fisher (1971b) SP 18, 253

Fisher (1971c) SP 19, 431

Fisher (1971d) SP 19, 436

Fisher (1972) SP 24, 385

Fisher, Pope (1971) SP 20, 389

Flower, Jordan, C. (1971) AA 14, 473

Fort et al. (1972) AA 17, 55

Foukal (1971) SP 19, 59

Foy (1972) SP 18, 26

Frazier (1970) SP 14, 89

Frazier (1971) SP 21, 42

Frazier (1972) SP 24, 98

Freeman, Jones (1970) SP 15, 288

Fullerton, Cowley (1971) AJ 165, 643

Gabriel (1971) SP 21, 392

Gabriel, Jordan, C. (1969) MN 145, 241

Gabriel et al. (1971) AJ 169, 595

Garstang (1971) JASWA 29, 2

Garz (1971) AA 10, 175

Garz et al. (1971) 2nd Conf. Atomic Spectroscopy,

Hannover, Germany 1970

Gay (1970a) AA 6, 327

Gay (1970b) AA 7, 24

Gibson, Van Allen (1970) AJ 161, 1135

Gingerich et al. (1971) SP 18, 347

Giovanelli (1970) PASA 1, 363

Giovanelli SP (P)

Giovanelli, Ramsey (1971) IAU 43, 293

Giovanelli et al. (1971) SP 22, 53

Gordon et al. (1971) AZ 48, 70

Grevesse, Swings (1970) SP 13, 19

Grevesse, Swings (1972) AJ 171, 179

Greve (1970) SP 15, 381

Greve (1971) SP 16, 328
Grigorev, Kuklin (1971) GGV Ser. 2 No. 13, 109

Grossman-Doerth, von Uexküll (1971) SP 20, 31

Grossman-Doerth, vion Uexküll (U)

Gulyaev (1971) SDB 10, 67

Gulyaev (1972) SP 24, 72

Gurtovenko, Alekayeva (1971) SP 21, 325

Gurtovenko, Alikayeva (1972) SP (P)

Hagen et al. (1971) SP 21, 286

Hall, Hinteregger (1970) JGR (Space Phys.) 75, 6959

Hall, Noyes (1972) AJ 175, L95

Hall et al. (1972) AJ 171, 3

Hansen, R. T., Garcia et al. (1969a) SP 7, 417

Hansen, R. T., Hansen, S. F. et al. (1969b) SP 10, 135

Hansen, R. T., Hansen S. F., Garcia (1970) SP 15,387

Hansen, R. T., Hansen, S. F. et al. (1971) SP 18, 271

Hansen, R. T., Hansen, S. F. et al. (1972) BAAS 4, 383

Hansen, S. F., Hansen, R. T. et al. (1972) BAAS 4, 383

Harvey (1972) NPS 235, 90

Harvey, Howard (1972) SP 23, 300

Harvey, Livingston (1970) AA 9, 151

Hata, Tojo (1972) ATAO Ser. 2, 13, 149

Hauge (1970) SP 11, 17

Hauge (1971) AA 10, 73

Héroux et al. (1972) SP 23, 369

Hiei, Hirayama (1970) PASJ 22, 545

High Altitude Observatory (1969) ChromosphereCorona Transition Region, NCAR, Boulder, Col.

High Altitude Observatory (1972) Line Formation in the Presence of a Magnetic Field, NCAR, Boulder, Col.

Holweger (1970) AA 4, 11

Holweger (1971) AA 10, 128

House (1972) SP 23, 103

Houtgast et al. (1972) SP 21, 281

Howard (1971) Solar Magnetic Fields, IAU 43, Reidel, Dordrecht

Howard, Stenflo (1972) SP 22, 402

Huang, Struve (1952) AJ 116, 410

James (1970) SP 12, 143

Janssens (1970) SP 11, 222

Jayanthan (1970) SP 12, 163

Jefferies (1969) 15 Colloque International d'Astrophys., Liège 1968, p. 213

Jefferies et al. (1971) SP 16, 103

Jefferies et al. (1972) SP 22, 307

Johnson (1971) BAAS 3, 385

Jones, Rense (1970) SP 15, 316

Jones et al. (1971) SP 21, 272

Jordan C. (1971) SP 21, 381 
Kaiser (1970) AJ 159, 77

Kandel, Keil (1971) BAAS 3, 376

Kanno et al. (1971) SP 21, 314

Karpinsky, Kostjukevich (1971) SDB 3, 88

Keller (1971) SP 21, 425

Kerimbekov (1971) SDB 4, 96

Kerimbekov et al. (1970) SDB 12, 88

Khersuriana et al. (1971) AZ 48, 985

Khlystov (1970) AZ 47, 103

Koomen et al. (1970) N226, 1138

Koutchmy (1971) AA 13, 79

Koutchmy (1972a) AA 16, 103

Koutchmy (1972b) SP 24, 373

Koutchmy, Peyturaux (1970) AA 5, 470

Koutchmy, Schatten (1971) SP 17, 117

Krat (1971) VANS 12, 18

Krat, Krat (1971) SP 17, 355

Krat, Stojanova (1971) SP 20, 57

Krieger, Barrett et al. (1972) BAAS 4, 386

Krieger, Paolini et al. (1972) SP 22, 150

Kumar (1970) Thesis, Univ. Michigan

Kundu (1971) SP 21, 130

Kundu, McCullough (1972) SP 24, 133

Labs, Neckel (1970) SP 15, 79

Labs, Neckel (1972) SP 22, 64

Lamb (1970) SP 12, 186

Lambert (1971) PTRSL A270, 3

Lambert et al. (1971a) SP 19, 289

Lambert et al. (1971b) MN 154, 265

Landman (1972) SP (P)

Landini, Monsignori Fossi (1971) SP 17, 379

Lantos (1972) SP 22, 387

Lantos-Jarry (1970) SP 15, 40

Lantos, Kundu (1971) AA 21, 119

Leblanc (1970) AA 4, 315

Leblanc et al. (1970) AA 5, 391

Lee et al. (1970) AO 9, 2653

Lena (1970) AA 4, 202

Leroy (1972a) AA 19, 287

Leroy (1972b) SP 25, 413

Leroy, Rosch (1970) SP 15, 383

Leroy et al. (1972) AA 17, 301

Lévy (1971) AA 14, 15

Lexa (1969) BAC 20, 373

Lexa (1971) BAC 22, 1

Linsky (1970) SP 11, 355

Linsky, Avrett (1970) PASP 82, 160

Linsky et al. (1970) SP 11, 374

Liu, Elske Smith (1971) SP 24, 301

Liu, Sheeley (1971) SP 20, 282

Liu et al. (1971) SP 23, 289

Livingston (1970) IAU Colloq. 1969, 321, Contr.

Kitt Peak National Obs. No. 531

Loughhead (1969) SP 10, 71

Loughhead, Tappere (1971) SP 19, 44

McDougal (1971) SP 21, 430
MacQueen et al. (1972) BAAS 4, 387

Macris (1971) Physics of the Solar Corona, Reidel, Dordrecht

Makarova et al. (1971) AZ 48, 360

Makita (1971) SP 24, 59

Matsushima, Kawabata (1972) AL 11, 103

Mehltretter (1971a) SP 16, 253

Mehltretter (1971b) SP 18, 510

Mewe (1972) SP 22, 459

Milkey (1970) SP 14, 62

Miller et al. (1970) BAAS 2, 331

Moore, Fung (1972) SP 23, 78

Mouradian (1967) SP 2, 258

Mouradian (1972) SP 24, 368

Mullan (1972) NPS 235, 58

Munro, Withbroe (1972) AJ (P)

Munro et al. (1971) SP 19, 347

Musman, Rust (1970) SP 13, 261

Nakagawa, Raadu SP 25, 127

Nakagawa et al. SP (P)

National Center for Atmospheric Research (1971) 1970 Ann. Rept. 49

National Science Foundation (1970) Solar Eclipse Bulletin, Washington D.C.

National Science Foundation (1973) Solar Eclipse Bulletin, Washington D.C.

Neupert (1971) SP 18, 474

Neupert et al. (1972) BAAS 4, 388

Newkirk (1971a) Physics of the Solar Corona, 66, Reidel, Dordrecht

Newkirk (1971b) IAU 43, 547, Reidel, Dordrecht

Newkirk (1972) Proc. Solar Wind Conf., Asilomar, NASA SP-308, 311.

Newkirk, Altschuler (1970) SP 13, 131

Newkirk, Harvey (1968) SP 3, 321

Newkirk et al. (1968) IAU 35, 369

Newkirk et al. (1970) SP 15, 15

Newkirk et al. (1972) SP 24, 370

Nikolsky (1970) SP 12, 379

Nikolsky et al. (1971) SP 21, 332

Noci (1972-73), preprint

Noyes (1971) ARAA 9, 209

Noyes, Kalkofen (1970) SP 15, 120

Noyes, Withbroe (1972) SSR (P)

Noyes et al. (1970) SP 11, 388

Noyes et al. (1972) SP (P)

O'Brien (1971) SP 19, 314

Olsen et al. (1971) SP 21, 360

Orrall (1972) SP 23, 30

Parker (1970) ARAA 8, 1

Parkinson, Pounds (1971) SP 17, 146

Parkinson, Reeves (1969) SP 10, 342

Parvey, Musman (1971) SP 18, 385

Pasachoff (1970) SP 12, 202

Pasachoff (1971) SP 19, 323 
Pasachoff, Zirin (1971) SP 18, 27

Pecker (1970) SP 15, 88

Pepin (1970) AJ 159, 1067

Perry, Altschuler (1972) SP (P)

Piddington SP (P)

Plaskett (1970) MN 148, 149

Pneuman (1969) SP 6, 255

Pneuman (1971) SP 19, 16

Pneuman (1972a) Proc. Solar Wind Conf., Asilomar, NASA SP-308, 55

Pneuman (1972b) SP 23, 223

Pneuman (1972c) AJ 177, 793

Pneuman, Kopp (1970) SP 13, 176

Pneuman, Kopp (1971) SP 18, 258

Pneuman, Raadu (1972) (P)

Porfirjeva (1971) AZ 48, 1227

Porfirjeva, Sitnik (1971) IKAO 41-42, 59

Prata (1971) SP 20, 30

Raadu (1972) SP 22, 443

Raadu, Nakagawa (1971) SP 20, 64

Ratier, Rozelot (1972) SP 23, 394

Rees (1971) SP 16, 67

Reeves et al. (1972) SP (P)

Reeves, Parkinson (1970) AJ Suppl. 181

Reeves, Parkinson (1972) SP 24, 113

Reiling (1971) SP 19, 297

Reza, Müller (1970) VSNG, 127

Reza, Müller (1971) A

Ribes, Unno (1971) PASA 2, 54

Rogers (1970) SP 13, 57

Rosenberg (1972) SP 25, 188

Ross, Aller (1970) PNAS 66, 983

Ross, Aller (1971) BAAS 3, 438

Roueff (1970) AA 7, 4

Rozelot (1972a) SP 22, 88

Rozelot (1972b) Adaptation de la Camera Electronique Lallemand au Coronagraph (P)

Saito (1970) ATAO, Ser. 2, 12, 53

Saito (1972) ATAO, Ser. 2, 13, 93

Saito, Hata (1970) ATAO, Ser. 2, 12, 151

Sarychev (1970) AZ 47, 1246

Sarychev (1971) AZ 48, 1232

Sawyer C., Hansen S. T. SP (P)

Scherrer et al. (1972) SP 22, 418

Schmidt et al. (1972) BAAS 4, 390

Schmieder (1972) AA 16, 44

Schoolman (1972) SP 22, 344

Semel (1970a) AA 5, 330

Semel (1970b) AA 9, 152

Semel (1970c) AA 9, 356

Sengupta (1971) SP 17, 160

Shatten (1972) Proc. Solar Wind Conf., Asilomar, NASA SP-308, 65

Shatten et al. (1968) SP 6, 442

Sheeley (1971) SP 20, 19

Sheeley, Bhatnagar (1971) SP 18, 195
Simon, G. W., Lynch (1972) BAAS 4, 391

Simon, G. W., Noyes (1971) IAU 43

Simon, G. W., Noyes (1972) SP 22, 450

Simon, M. (1971) SP 21, 297

Sitnik et al. (1970) AZ 47, 516

Slaughter, Wilson (1972) SP 24, 43

Smerd, Dulk (1971) IAU 43, Reidel, Dordrecht

Smith, Suffolk (1972) BAAS 4, 392

Snider (1972) PRL 28, 853

Sonett et al. (1972) Proc. Solar Wind Conf., Asilomar, NASA SP-308

Sotirovski (1972) AA 6, 85

Speer et al. (1971) N 226, 249

Stanek (1972) SP 21, 121

Stanek (1972) AMZ 309

Stellmacher, Wiehr (1971) SP 18, 220

Stettler et al. (1971) ASHSN 45

Stelzried et al. (1970) SP 14, 44

Svensson (1971) SP 18, 232

Swensson et al. (1970) MSRSL, Sp. Vol. 5, 40

Sykora (1971) SP 18, 72

Tanaka (1971) PASJ 23, 185

Tanaka, Hiei (1972) PASJ 24, 323

Thiessen (1955) ZA 35, 237

Third Symposium on Ultraviolet and $X$-Ray Spectroscopy of Astrophysical and Laboratory Plasmas, Utrecht (P)

Thomas (1972) SP 24, 262

Timothy et al. (1972) BAAS 4, 393

Tousey (1971) PTRSL A270, 59

Tousey, Koomen (1971a) SP 21, 401

Tousey, Koomen (1971b) BAAS 4, 394

Travis, Matsushima (1968) AJ 154, 689

Trotter, Newkirk (1971) SP 20, 372

Tsubaki et al. (1971) SP 21, 305

Tucker, Koren (1971) AJ 168, 283

Uchida (1970) PASJ 22, 341

Underwood, Neupert (1972) BAAS 4, 394

Unsöld (1971) PTRSL 270, 23

Van Dessel (1970) SP 15, 322

Van Speybroeck et al. (1970) N 227, 818

Vardya (1972) AJ 171, 185, \& priv. comm.

Veeder, Zirin (1970) SP 12, 391

Vernazza, Noyes (1972) SP 22, 358

Wagner, House (1971) AJ 166, 683

Waldmeier (1970) SP 15, 167

Waldmeier (1971a) AMZ 307

Waldmeier (1971b) Physics of the Solar Corona, 130, Reidel, Dordrecht

Waldmeier (1971c) SP 30, 332

Waldmeier, Weber (1971) AMZ 308

Walker, Rugge (1972) BAAS 4, 395

White SP (P)

White, Bhavilai (1970) AL 5, 137 
White et al. (1972) SP 23, 18

Widing et al. (1970) SP 12, 52

Widing et al. (1971) AJ 169, 405

Wijbenga, Zwaan (1972) SP 23, 265

Wilson, Evans (1971) SP 18, 29

Wilson et al. (1972) SP 25, 86

Withbroe (1970a) SP 11, 42

Withbroe (1970b) SP 11, 208

Withbroe (1971a) SP 18, 458
Withbroe (1971b) The Menzel Symposium, NBS Spec. Publ. 353, 127

Withbroe et al. (1971) SP 21, 272

Wolnik et al. (1970) AJ 162, 1037

Wolnik et al. (1971) AJ 166, L31

Worrall (1971) AA 12, 88

Zirin (1970) SP 11, 497

Zirin (1972) SP 22, 34

Zirker (1970) SP 11, 68 